\title{
An Improved Linear Model for High Frequency Class-DE Resonant Converter using the Generalized Averaging Modeling Technique
}

Dahl, Nicolai Jerram; Ammar, Ahmed Morsi; Knott, Arnold; Andersen, Michael A. E.

Published in:

IEEE Journal of Emerging and Selected Topics in Power Electronics

Link to article, DOI:

10.1109/JESTPE.2019.2945182

Publication date:

2020

Document Version

Peer reviewed version

Link back to DTU Orbit

Citation (APA):

Dahl, N. J., Ammar, A. M., Knott, A., \& Andersen, M. A. E. (2020). An Improved Linear Model for High

Frequency Class-DE Resonant Converter using the Generalized Averaging Modeling Technique. IEEE Journal of Emerging and Selected Topics in Power Electronics, 8(3), 2156 - 2166.

https://doi.org/10.1109/JESTPE.2019.2945182

\section{General rights}

Copyright and moral rights for the publications made accessible in the public portal are retained by the authors and/or other copyright owners and it is a condition of accessing publications that users recognise and abide by the legal requirements associated with these rights.

- Users may download and print one copy of any publication from the public portal for the purpose of private study or research.

- You may not further distribute the material or use it for any profit-making activity or commercial gain

- You may freely distribute the URL identifying the publication in the public portal 


\title{
An Improved Linear Model for High Frequency Class-DE Resonant Converter using the Generalized Averaging Modeling Technique
}

\author{
Nicolai J. Dahl, Student Member, IEEE, Ahmed M. Ammar, Student Member, IEEE, Arnold Knott, \\ Michael A. E. Andersen, Member, IEEE
}

\begin{abstract}
As the operating frequency of power converters increases, the passive component values likewise decrease. This results in the effect of the parasitic components becoming more prominent, leading to significant modeling errors if not considered. For resonant converters this especially becomes a problem at high frequencies. This paper presents a reduced model for a class-DE series resonant converter based on generalized averaging that incorporates the relevant parasitics and uses multiple harmonics to obtain an accurate linear model. Comparison between the proposed model, prior art, and a prototype converter running at $1 \mathrm{MHz}$ is conducted, and a PI-controller is designed based on each model and tested. The results show that the parasitics have a significant impact on the DC-gain and dynamics of the converter, and that the proposed model improves on the prior art by reducing the DC-gain error by more than $7 \mathrm{~dB}$, and the error in the low frequency pole from $168 \%$ to 16.9 $\%$. Furthermore, the PI-controller designed on the prior art was found to have more than 40 times larger overshoot in the control signal when measured compared to the model prediction, while the controller based on the proposed model showed correct performance when simulated and measured.
\end{abstract}

Index Terms-Resonant converters, Modeling, PI control, State-space methods, DC-DC power converters.

\section{INTRODUCTION}

$\mathbf{T}$ ODAY, most electronic devices are powered with a Switch Mode Power Supply (SMPS) due to their small form factor and high efficiency. The majority of the SMPS are different types of hard switching PWM topologies. These supplies have high switching losses which limit the switching frequency that the supplies can handle. Nonetheless, higher switching frequencies allow for smaller passive components, and hence an overall smaller power supply, as well as higher bandwidth to better react to various load and line disturbances. Therefore, to improve on the switching frequencies, soft switching topologies have been receiving much attention in recent years [1-7]. One family of soft switching converters are the resonant converters [8]. Resonant converters work by having a resonant tank take care of charging and discharging the switching node before the switching event, and thus obtain Zero Voltage Switching (ZVS). This vastly reduces the switching losses, allowing for a higher operation frequency.

This project has received funding from the European Union's Horizon 2020 research and innovation programme under grant agreement No. 731466 The authors are with the Department of Electrical Engineering, Technical University of Denmark, Kgs. Lyngby (e-mail: nicoje@elektro.dtu.dk; ammma@elektro.dtu.dk; akn@elektro.dtu.dk; ma@elektro.dtu.dk)
The typical design and modeling procedures used for PWM converters do not apply to resonant converters. PWM converter models are fundamentally built on the small ripple hypothesis, meaning that the ripple can be averaged and only the underlying linear dynamics need to be considered $[9,10]$. This is not applicable with resonant converters as it would average out the resonance in the resonance tank to zero. To resolve this, models that are able to capture the periodic behavior have been developed. One modeling approach is to model the converter using multiple subsystems connected with a reset map [11-13]. This method maintains all the information of the converter, but is not very pratical for most analysis and control design. Another modeling approach which has recieved a lot of attention in the modeling of active front ends is the Linear Time-Varying Periodic (LTP) model [14-18]. This method uses a harmonic state space model to descripe how each of the harmonic components of the input impact each of the harmonic components of the output. Lastly, there are the modeling approaches based on the generalized averaging model [19-22]. This modeling approach extends the original averaging model method by describing the periodic behavior of the system using a Fourier series. Many of these modeling approaches come with a set of assumptions to simplify the problem. These assumptions are typical about purely sinusoidal signals or fast decaying harmonics, resulting in solutions which are only valid in a limited range if not fulfilled. Furthermore, the prior art, that uses the modeling techniques presented above, only considers converters operating at frequencies up to $200 \mathrm{kHz}$. At these frequencies, the parasitic components are negligible, and for that reason not included in the models. However, with the increase in operating frequency, this is no longer the case and the models breaks down.

This paper presents a model of a class-DE Series Resonant Converter (SRC) where the effect of the parasitics is included to make the model valid for high-frequency operation. The model is based on the generalized averaging method, and considers multiple harmonics to obtain a precise representation of the converter behavior. The resulting model is of a high-order, so a model reduction approach is applied to simplify the model to a low order, high precision, and computational robust linear model which is valid for a wide operation range. The proposed reduced model is validated against a $1 \mathrm{MHz}$ class-DE SRC and compared to prior art. Lastly, a PI-controller is designed 
based on both the proposed model and prior art, and tested to assess the performance improvement. Although the presented model is for a class-DE SRC, the presented modeling method with the same parasitics can also be used to model variations of the class-DE resonant network, like the LLC, LCC, and parallel resonant converter.

\section{LARGE SignAl MODEL}

Using Kirchoff's current and voltage laws, the discontinuous differential equations governing the behavior of the class-DE converter shown in Fig. 1 are established. Equation (1) shows the derived equations which are similar to the findings in [21], but also include the equivalent series resistance for the capacitor and inductor, here lumped together in $R_{e s r}$ (not shown in the circuit).

$$
\begin{aligned}
\dot{I}_{r} & =\frac{V_{s w}}{L_{r}}-\frac{V_{r}}{L_{r}}-I_{r} R_{e s r}-\frac{V_{h b}}{L_{r}} \\
\dot{V}_{r} & =\frac{I_{r}}{C_{r}} \\
\dot{V}_{o} & =\frac{I_{h b}}{C_{f}}-\frac{V_{o}}{C_{f} R_{f}}
\end{aligned}
$$

where $I_{r}$ is the periodic current in the resonance tank, $V_{r}$ is the voltage across the resonance tank capacitor $C_{r}$, and $V_{o}$ is the output voltage of the converter. $V_{s w}, V_{h b}$, and $I_{h b}$ are the switching node voltage, the voltage at the rectifier, and the current through the rectifier to the output, respectively, and are all piece-wise continuous functions. In reported work [20, 21] these piece-wise continuous functions are assumed to be ideal, e.g. the effect of parasitic components is disregarded. This is a fair assumption for medium frequency operation $(<500 \mathrm{kHz})$ where it provides satisfactory results. However, as the switching frequency is increased, the capacitor and inductor sizes in the resonance tank decrease and the parasitic components of the switching devices start to become significant and interact with the converter. The parasitics, among other factors, cause a change in the resonance frequency and delaying of charge transfer in the rectifier. Therefore, to obtain an accurate model of the converter at high frequency switching, the relevant parasitics need to be identified and included in the model.

The dominant parasitics come from the switching devices in the converter, i.e. the FETs and the diodes, and are capacitive by nature. Thus the output capacitance of the FETs, $C_{o s s}$, and the capacitance of the diodes, $C_{d}$ are added to the model. Both of these capacitors are nonlinear dependent of the voltage across the device, and will change in value during charging/discharging. Hence, the capacitor values are approximated by the time related capacitors to give an equivalent behavior. The parasitics in the resonant tank are, in the $\mathrm{MHz}$ range, usually insignificant in size, and thus not considered in the model. Fig. 1 shows the class-DE series resonant converter with the relevant parasitics.

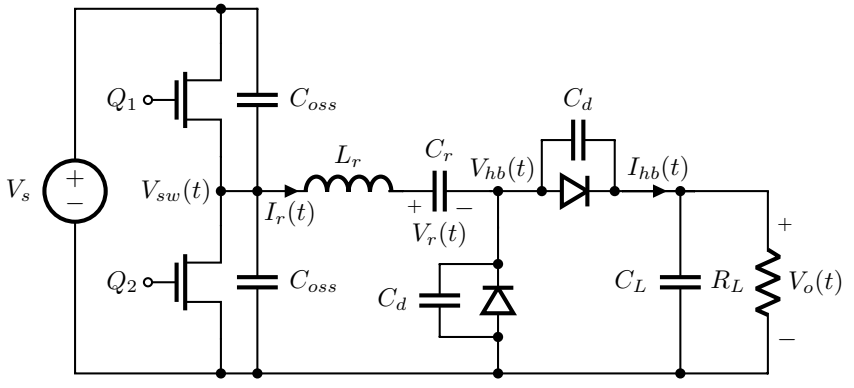

Fig. 1: The class-DE series resonant converter with the parasitic capacitance for the FET's and diodes. The ESR resistances of $L_{r}$ and $C_{r}$ are not explicitly shown in the circuit, but still included in the calculations.

Looking at Fig. 1 it is seen that all the parasitic components are connected to nodes that are discontinuous and hence need to be described by piece-wise functions. To work out the exact behavior of these piece-wise functions, a transient simulation of the converter is performed. Fig. 2 shows the steady state operation of the discontinuous nodes $V_{h b}, I_{h b}$ and $V_{s w}$ in normalized time.
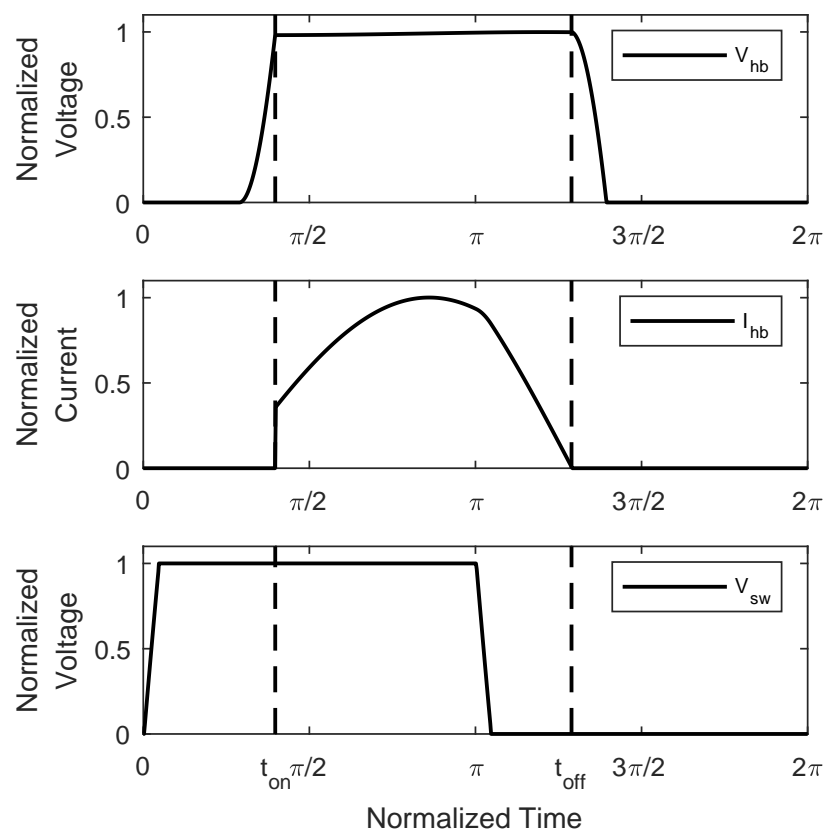

Fig. 2: The discontinuous waveforms of the converter. The dashed lines indicate the conduction time of the rectifier.

From Fig. 2, the effects of the added parasitics are observed. Once the resonance current $I_{r}$ becomes positive (about $\pi / 4$ ), the diode capacitance $C_{d}$ starts charging hence increasing the voltage in $V_{h b}$. This prevents the charge transfer in the rectifier to the output since the forward voltage of the diodes is yet to be reached. Then, when $V_{h b}=V_{o}$ the diode can start conducting and will keep on conducting until the resonance current becomes negative. At that point, the diode capacitance will be discharging. In the ideal case, $V_{h b}$ would ramp up instantaneously, allowing the current to be passed to the output for the entire positive half-cycle. However, due to the parasitic 
capacitance in the rectifier diodes, this is no longer the case.

Regarding the switching node, $V_{s w}$, it resembles a square waveform, however, with a small slope on the edges. The slopes come from the charging and discharging of the $C_{o s s}$ during the dead time of the gate signals when none of the FETs are conducting. This leads to the system being in its unforced stage, and the resonance current will charge/discharge $C_{\text {oss }}$. The length of the dead time is given by $\pi(1-2 D)$ where $D$ is the positive duty cycle of the gate signals to the FETs. Ideally, it is desired to tune the duty cycle such that $C_{o s s}$ is charged to $V_{s}$ and zero voltage switching occurs. In case $C_{o s s}$ is over charged, diodes in parallel with $C_{o s s}$ will start conducting, limiting the voltage. However, these diodes are not included in Fig. 1 and neither in the presented model since it would lead to $V_{s w}$ being piece-wise in both time and voltage. Functions that are piece-wise in more than one variable are increasingly difficult to model and approximate as the problem becomes multidimensional. Hence the complete model is only valid in operation modes where these diodes are not conducting, i.e. partial and full ZVS. In operation modes where the diodes are conducting, the model will over charge the voltage in the switching node, $V_{s w}$ to maintain the resonant current. Thus the resonant current and output voltage remain valid in this operation mode, and only $V_{s w}$ will differ from the expected behavior.

From the behavior described above the piece-wise equations are formalized. Equation (2) to (4) shows the piece-wise functions. Each equation is periodic, i.e. $f(t)=f(t+2 \pi)$, and $\phi$ represents the phase shift observed in Fig. 2 .

$$
\begin{gathered}
V_{h b}(t-\phi)= \begin{cases}\frac{\int I_{r} \mathrm{~d} t}{2 C_{d} \omega} & 0 \leq t<t_{\text {on }} \\
V_{o} & t_{\text {on }} \leq t<\pi \\
\frac{\int I_{r} \mathrm{~d} t}{2 C_{d} \omega}+V_{o} & \pi \leq t<\pi+t_{\text {on }} \\
0 & \pi+t_{\text {on }} \leq t<2 \pi\end{cases} \\
I_{h b}(t-\phi)= \begin{cases}0 & 0 \leq t<t_{\text {on }} \\
I_{r} & t_{\text {on }} \leq t<\pi \\
0 & \pi \leq t<2 \pi\end{cases} \\
V_{s w}(t)= \begin{cases}\int-I_{r} \mathrm{~d} t & 0 \leq t<\pi(1-2 D) \\
\frac{V_{s} C_{o s s} \omega}{V_{-I_{r} \mathrm{~d} t}^{2 C_{o s s} \omega}+V_{s}} & \pi \leq t<2 \pi(1-D) \\
0 & 2 \pi(1-D) \leq t<2 \pi\end{cases}
\end{gathered}
$$

where $\omega$ is the switching frequency $f_{s w}$ in $\mathrm{rad} / \mathrm{sec}$, and $t_{\text {on }}$ is the point where $I_{h b}$ becomes non-zero. If $\omega$ is assumed to be constant, the equations become piece-wise linear. $t_{o n}$ is found as the time it takes to charge both $C_{d}$ to $V_{o}$ using a sinusoidal current with magnitude $I_{m}$. Thus the calculation of $t_{\text {on }}$ assumes an ideal resonance current. Equation (5) shows the function for $t_{o n}$.

$$
t_{\text {on }}=\arccos \left(1-\frac{2 \omega C_{d} V_{o}}{I_{m}}\right)
$$

With (1) through (5), we obtain a complete piece-wise mathematical representation of the converter with the described parastics.

\section{HARMONIC APPROXIMATION}

The mathematical model developed in section II can be expressed as multiple models, all connected through switching surfaces [12]. This allows for a precise discontinuous description of the system which is suited for simulation. However, the model is impractical for further analysis and control design where a single Linear Time Invariant (LTI) model is preferred. Thus the model needs to be linearized. The resonance converter exhibits a limit cycle in its steady-state operation, making the conventional averaging linearization methods inapplicable, and other more generalized methods are needed. One such method, and the method used in prior art, is the harmonic balance method. This method approximates each state with a Fourier series, where the magnitude for each term becomes the new state of the system. The approximation will result in a loss of information as the Fourier series will at some point be truncated. Equation (6) shows the approximation of the states by the Fourier series to the arbitrary order $N$. Equation (7) shows the same but for the state derivatives.

$$
\begin{gathered}
I_{r} \approx I_{r_{D C}}+\sum_{n=1}^{N} I_{r_{s n}} \sin (n \omega t)+I_{r_{c n}} \cos (n \omega t) \\
V_{r} \approx V_{r_{D C}}+\sum_{n=1}^{N} V_{r_{s n}} \sin (n \omega t)+V_{r_{c n}} \cos (n \omega t) \\
V_{o} \approx V_{o_{D C}}+\sum_{n=1}^{N} V_{o_{s n}} \sin (n \omega t)+V_{o_{c n}} \cos (n \omega t) \\
\dot{I}_{r} \approx \dot{I}_{r_{D C}}+\sum_{n=1}^{N}\left(\dot{I}_{r_{s n}}-\omega n I_{r_{c n}}\right) \sin (n \omega t)+ \\
\dot{V}_{r} \approx \dot{V}_{r_{D C}}+\sum_{n=1}^{N}\left(\dot{I}_{r_{c n}}+\omega n I_{r_{s n}}\right) \cos (n \omega t) \\
\left.\dot{V}_{r_{s n}}-\omega n V_{r_{c n}}\right) \sin (n \omega t)+ \\
\left(\dot{V}_{r_{c n}}+\omega n V_{r_{s n}}\right) \cos (n \omega t) \\
+\sum_{n=1}^{N}\left(\dot{V}_{o_{s n}}-\omega n V_{o_{c n}}\right) \sin (n \omega t)+ \\
\left(\dot{V}_{o_{c n}}+\omega n V_{o_{s n}}\right) \cos (n \omega t)
\end{gathered}
$$

With the approximation of the states, the state vector goes from being of order 3 to order $3+6 N$, where $N$ is the order of the Fourier series. Equation (8) shows the new state vector. Here $I_{r_{c n}}, I_{r_{s n}}$, etc. are vectors of length $N$ containing the states for each harmonic. 


$$
x=\left[\begin{array}{l}
I_{r} \\
V_{r} \\
V_{f}
\end{array}\right] \mapsto\left[\begin{array}{c}
I_{r_{D C}} \\
V_{r_{D C}} \\
V_{o_{D C}} \\
I_{r_{c n}} \\
V_{r_{c n}} \\
V_{o_{c n}} \\
I_{r_{s n}} \\
V_{r_{s n}} \\
V_{o_{s n}}
\end{array}\right]
$$

Besides the states, the piece-wise functions (2)-(4) each needs to be approximated by a single continuous function. Since the piece-wise functions are periodic, a Fourier series can likewise be used. The Fourier series are calculated through conventional methods, and then rewritten to simplify the computation. The Appendix presents the used rewritten Fourier series. Fig. 3 shows the normalized magnitudes of the Fourier coefficients for each of the constructed series.

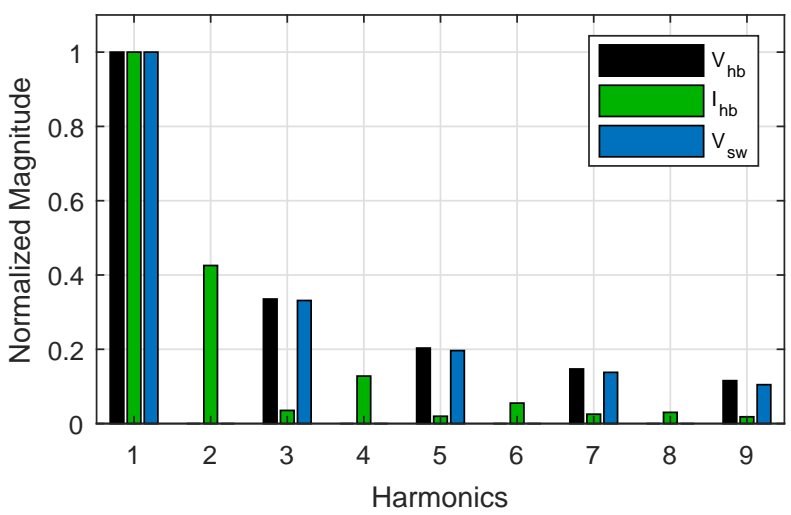

Fig. 3: Normalized magnitudes of the harmonic content for each Fourier series.

Fig. 3 shows that $V_{h b}$ and $V_{s w}$ are described purely by the odd harmonics in the Fourier series. This results in all the even terms, except zero, in the states $I_{r_{c n}}, I_{r_{s n}}, V_{r_{c n}}$, and $V_{r_{s n}}$ evaluate to zero and the states can be discarded as they provide no information. This reduces the size of the system to $3+4 N$ for an even $N$. Furthermore, from Fig. 1 it can also be concluded that $I_{r_{D C}}$ will always be zero in steady state due to the resonance tank capacitor $C_{r}$ blocking DC current flow. However, $I_{r_{D C}}$ is non-zero during transients. Thus it still provides some information.

\section{A. Harmonic Balancing}

With the Fourier approximation of both the states and the piece-wise functions completed, the approximations are inserted in (1). This creates a set of partial differential equations (PDEs). However, by collecting the trigonometric terms, the partial differential equation can be separated into a set of first order ordinary differential equations (ODEs), where the trigonometric term can be canceled out. Equation (9) shows the resulting three equations for (1c) when the described method is applied. Here a 1st order Fourier series is used, and $I_{h b_{D C}}, I_{h b_{s}}$, and $I_{h b_{c}}$ are the calculated Fourier coefficients.

$$
\begin{aligned}
\dot{V}_{o_{D C}} & =\frac{I_{h b_{D C}}}{C_{f}}-\frac{V_{o_{D C}}}{C_{f} R_{f}} \\
\dot{V}_{o_{s}} & =\frac{I_{h b_{s}}}{C_{f}}-\frac{V_{o_{s}}}{C_{f} R_{f}}+\omega V_{o_{c}} \\
\dot{V}_{o_{c}} & =\frac{I_{h b_{c}}}{C_{f}}-\frac{V_{o_{c}}}{C_{f} R_{f}}-\omega V_{o_{s}}
\end{aligned}
$$

The Fourier coefficients will be nonlinear expressions dependent on one or more of the states, making the system of firstorder ODEs nonlinear. Due to the Fourier series, the resulting system is a continuous approximation of the discontinuous system in (1) where the discontinuities have been eliminated at the expense of an increased number of states. The nonlinear approximation obtained from the Fourier series (17)-(19) is not accurate in transient simulations due to simplifications. However, other realizations of the Fourier series will result in accurate transient simulations but at the expense of increased computation time. Nonetheless, the operating point for all the states of the resonant converter can be determined by inserting the desired duty cycle and frequency into the system, and solve for the steady-state using a numerical solver.

\section{Linear State Space Model}

With the nonlinear approximation model constructed and a suitable operation point found, linearization of the model is done to obtain a state space model of the form

$$
\begin{aligned}
& \dot{x}=A x+B u \\
& y=C x
\end{aligned}
$$

where $A$ is the system matrix, $B$ is the input matrix, and $C$ is the output matrix. The state vector $x$ will contain the Fourier expanded variables as presented in (8). Since the even harmonics of the resonant voltage and current turned out to be redundant, the differential equations describing these can be removed prior to linearization without any loss of information. The input vector $u$ consist of the switching frequency, $f_{s w}$, the duty cycle, $D$, and the supply voltage, $V_{s}$, where the latter is used to include potential disturbances from the supply. Lastly, the output vector $y$ consists of the DC output voltage, $V_{o_{D C}}$. The obtained state space model will only be valid up to the Nyquist frequency due to the selected modeling approach. Nonetheless, this still results in an accurate model for controller design and general analysis. For cases where dynamic content above the Nyquist frequency is needed, the modeling approach presented in [23] can be used. This modeling method provides an extended range of the model but at the expense of beings computationally more expensive.

\section{A. Model Reduction}

The created state space model contain both a large portion of zero elements and some large values in the system matrix. This leads to a high condition number of the model, indicating that the model has poor numerical properties and may produce numerical errors in further computations. Furthermore, because 
of the large size of the state space model, the model is not practical for control design. Also, conversion to transfer functions are discouraged due to the reduction of the numerical precision inherent in the conversion process. Thus to address these problems, the state space model needs to be adjusted and reduced in order to maintain reliability for later computations. The reduction is also necessary from a control perspective where a smaller number of states needs to be considered, making observer designs and complex control strategies simpler and faster to implement and execute.

One procedure to prepare the system for a model reduction that also tend to improve the condition number of the state space model is to balance the system. A balanced system is a system where the states are selected in such a way that each state is as controllable as it is observable. Then the relative impact of each state is given by the Hankel Singular Values (HSV). The HSV is defined as $\lambda^{\frac{1}{2}}(P Q)$, where $P$ and $Q$ are the controllability and observability Gramians respectively. For linear systems $P$ and $Q$ can be determined using the two Lyapunov equations in (11) [24, 25].

$$
\begin{aligned}
& A P+P A^{T}+B B^{T}=0 \\
& A^{T} Q+Q A+C^{T} C=0
\end{aligned}
$$

If $P$ and $Q$ are both diagonal matrices containing the HSVs sorted from highest to lowest, the states in $A$ are balanced and, likewise, sorted. Hence the first states are those with the largest impact on the system dynamics down to the last state which has the least impact. Moreover, all the elements in the matrices will be non-zero, and the numerical values will be closer, thereby improving the condition number.

With the system balanced, model reduction can be achieved easily. Since the states are already sorted by their dynamical impact, the state space system can be partitioned into the parts to keep, denoted $\left(A_{11}, B_{1}, C_{1}\right)$, and the parts to discard, as shown in (12). The exact number of states to keep can usually be determined by inspecting the HSVs and observing if any state has a large decrease in its HSV. Lastly, the reduced model is derived using a residualization based reduction (13). The residualization technique sets $\dot{x}_{2}=0$, thereby assuming steady state, and solves for $x_{2}$ in terms of $x_{1}$ and $u$. The result is backsubstituted into the equation for $x_{1}$ resulting in the matrices in (13). This method ensures that the information regarding the DC-gain in the discarded states is preserved in the reduced model thus improving the overall precision at low frequencies which is critical for power converters.

$$
\begin{aligned}
& {\left[\begin{array}{l}
\dot{x}_{1} \\
\dot{x}_{2}
\end{array}\right]=\left[\begin{array}{ll}
A_{11} & A_{12} \\
A_{21} & A_{22}
\end{array}\right]\left[\begin{array}{l}
x_{1} \\
x_{2}
\end{array}\right]+\left[\begin{array}{l}
B_{1} \\
B_{2}
\end{array}\right] u} \\
& {\left[\begin{array}{l}
y_{1} \\
y_{2}
\end{array}\right]=\left[\begin{array}{ll}
C_{1} & C_{2}
\end{array}\right]\left[\begin{array}{l}
x_{1} \\
x_{2}
\end{array}\right]}
\end{aligned}
$$

$$
\begin{aligned}
& A_{r}=A_{11}-A_{12} A_{22}^{-1} A_{21} \\
& B_{r}=B_{1}-A_{12} A_{22}^{-1} B_{2} \\
& C_{r}=C_{1}-C_{2} A_{22}^{-1} A_{21}
\end{aligned}
$$

\section{EXPERIMENTAL VALIDATION}

To verify the presented theory, we construct a class-DE SRC with the specifications and bill of material presented in Table I. These values are also used in the model such that a comparison with the implemented converter can be made.

TABLE I: Test conditions of the converter and the corresponding bill of materials.

\begin{tabular}{c|clc}
\hline & Value & Unit & Acquired \\
\hline Test Conditions & & & \\
$V_{s}$ & 348 & $\mathrm{~V}$ & Measured \\
$V_{o}$ & 210 & $\mathrm{~V}$ & Measured \\
$D$ & 47.9 & $\%$ & Known \\
$f_{s w}$ & 1.01 & $\mathrm{MHz}$ & Known \\
$f_{r}$ & 865 & $\mathrm{kHz}$ & Calculated \\
& & & \\
Bill of Materials & & & \\
$L_{r}$ & 150 & $\mu \mathrm{H}$ & Measured \\
$C_{r}$ & 240 & $\mathrm{pF}$ & Estimated \\
$C_{\text {oss }}$ & 53 & $\mathrm{pF}$ & Datasheet [26] \\
$C_{d}$ & 20 & $\mathrm{pF}$ & Datasheet [27] \\
$R_{e s r}$ & 1.5 & $\Omega$ & Measured \\
$R_{L}$ & 1000 & $\Omega$ & Known \\
$C_{L}$ & 30 & $\mathrm{nF}$ & Measured \\
\hline
\end{tabular}

For the resonance tank capacitor, $C_{r}$, a $220 \mathrm{pF}$ capacitor with a $10 \%$ tolerance is used. However, the value is increased slightly to account for parasitic capacitance in the resonance tank. For $C_{\text {oss }}$ and $C_{d}$, the capacitance is found from the datasheets by calculating the average capacitance for the voltage range each device experiences.

\section{A. Deriving the Model}

Using the specifications in Table I and the theory presented in section III, an approximative nonlinear dynamical model using a 5th order Fourier series is constructed. A Fourier series of order 5 is found to be a good compromise between sufficient convergence of the Fourier series and needed computation time. For orders lower than 5, the precision of the model decreases rapidly, while for higher orders, the numerical issues become worse without adding much to the precision. To illustrate the increase in precision with the order of the Fourier series, the operation point is calculated for every second order going from order 1 through 9. Table II shows the computed DC output voltage, $V_{O_{D C}}$ for each operation point with the stepwise change in the result, $\Delta V$. The stepwise change illustrates the convergence of the model for the given order of the Fourier series, by considering the difference in the output voltage between the models. 
TABLE II: Computed output voltage for different order Fourier series. $\Delta V$ shows the change in the output voltage in volt and percent between the associated order model and the model to the left.

\begin{tabular}{c|ccccc}
\hline Order & 1 & 3 & 5 & 7 & 9 \\
\hline$V_{O_{D C}}$ & $220.57 \mathrm{~V}$ & $212.55 \mathrm{~V}$ & $212.44 \mathrm{~V}$ & $212.42 \mathrm{~V}$ & $211.92 \mathrm{~V}$ \\
$\Delta V$ & & $-8.02 \mathrm{~V}$ & $-0.11 \mathrm{~V}$ & $-0.02 \mathrm{~V}$ & $-0.5 \mathrm{~V}$ \\
& & $-3.6 \%$ & $-0.052 \%$ & $-0.009 \%$ & $-0.234 \%$ \\
\hline
\end{tabular}

The computed operation point is inserted into the state space model to obtain the linear model. The resulting model is a 25 states model with a condition number of $1.5 \cdot 10^{6}$. By balancing the model, the condition number is reduced to 855 thus providing a numerical improvement of roughly 3 decimals. Finally, the model reduction is performed using the method described in Section IV-A. The HSVs are extracted to determine each states impact on the dynamics of the converter. Fig. 4 shows the value of the HSV related to each state in the balanced high-order state space model.

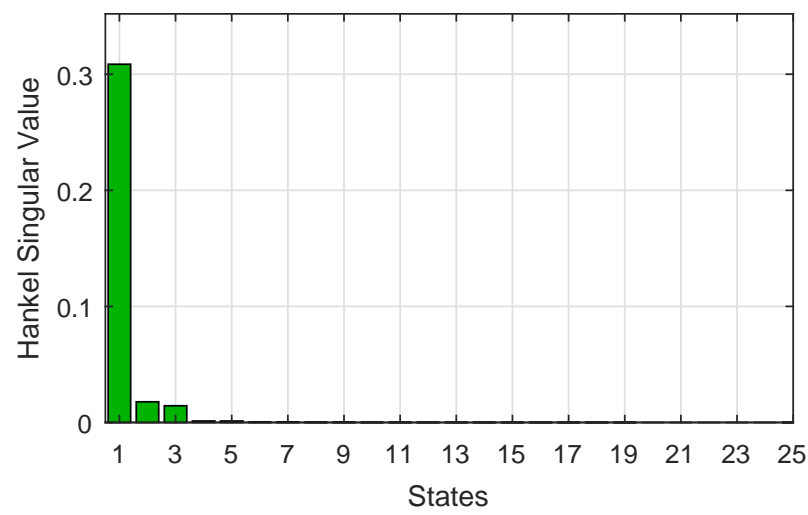

Fig. 4: The Hankel Singular Values corresponding to each state of the balanced high-order state space model of the resonant converter.

A large drop in the HSV is observed when going from 1 state to 2 states and when going from 3 states to 4 states. Above 4 states, the HSVs are so small that the dynamical impact is negligible. This suggest that a 3 rd order model is sufficient to describe the behavior of the converter. Equation (14) shows the reduced 3rd order state space model of the system.

$$
\begin{aligned}
A_{r} & =\left[\begin{array}{ccc}
-4.12 & -1.08 & -10.2 \\
-1.21 & -1.78 & 104 \\
10.2 & -103 & -69.3
\end{array}\right] 10^{4} \\
B_{r} & =\left[\begin{array}{cc}
0.214 & -160 \\
0.171 & -26.0 \\
-0.239 & 157
\end{array}\right] \\
C_{r} & =\left[\begin{array}{ccc}
-160 & -23.7 & -157
\end{array}\right]
\end{aligned}
$$

To ensure that the reduction removes only non-essential state information, the frequency and time information between the high-order model and the reduced model is compared. Fig. 5 shows the frequency response for the reduced and high-order model, and Fig. 6 shows the step responses.

Looking at Fig. 5 we find that the DC and low frequency content is preserved in the reduced model thanks to the residualization method used. From $800 \mathrm{kHz}$ and above, the high-order model contains information regarding the switching behavior which is not preserved in the reduced model. Since the converter operates with a switching frequency of $1 \mathrm{MHz}$, the reduced model is more than adequate to describe the low frequency dynamics of the converter. This is also clearly illustrated in the step responses in Fig. 6 where the reduced model precisely matches the high-order model. The reduction of the model further reduces the condition number down to 33.

\section{B. Measurements}

Fig. 7 shows the implemented class-DE converter prototype that has been tested in the laboratory. The switching frequency of the prototype is controlled by a voltage controlled oscillator (VCO). To get a good comparison between the models, the prototype, and existing models, multiple measurements are conducted. First, the steady state output voltage is obtained

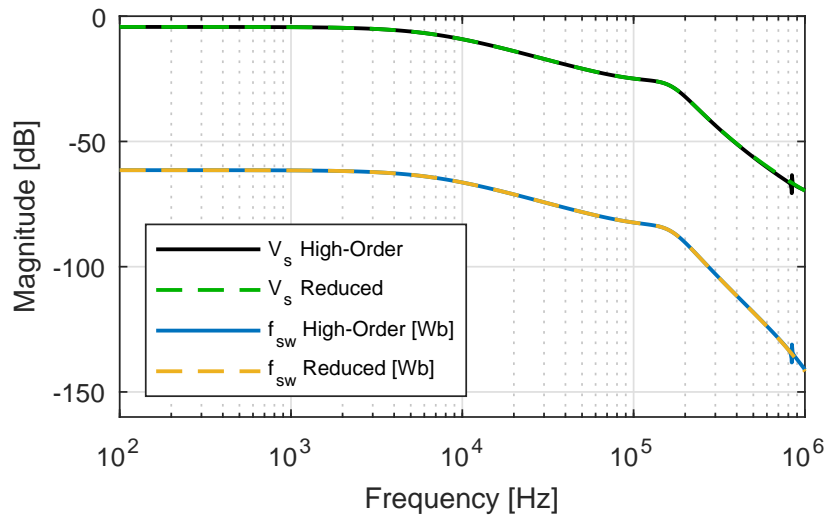

Fig. 5: Calculated frequency response of the high-order and the reduced state space models for the switching frequency, $f_{s w}$, and the supply voltage, $V_{s}$, to the output, $V_{o}$.

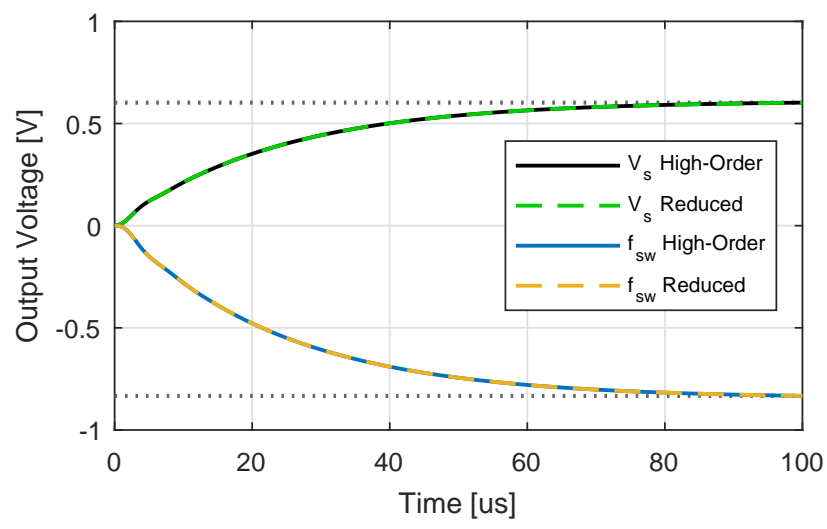

Fig. 6: Calculated step response of the high-order and the reduced state space models. The switching frequency, $f_{s w}$, is stepped $1 \mathrm{kHz}$ and the supply voltage, $V_{s}$, is stepped $1 \mathrm{~V}$. All signals are offset to zero. 


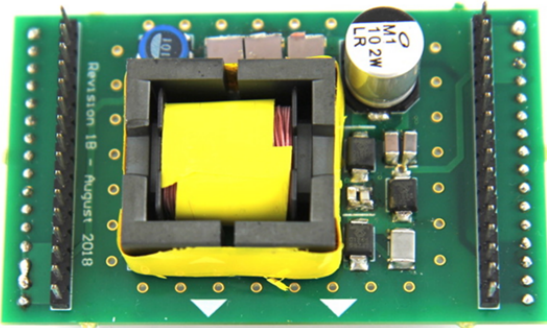

Fig. 7: The implemented Class-DE SR Converter.

for the prototype, the proposed model, and prior art [20, 21]. Second, measurements of the frequency response from the switching frequency to the output voltage is taken for the prototype and calculated for the models. The frequency response is measured using a frequency response analyser which superimposes a small sinosoidal signal on the input of the VCO through an injection transformer. The input voltage of the VCO and the output voltage of the prototype are measured from where the frequency response is calculated.Lastly, step responses from both the supply voltage and the switching frequency to the output voltage are conducted. For the step responses, all the steps are offset to $0 \mathrm{~V}$ at $t=0$ to better compare the transient behavior and the gain. The prior art with which the proposed model is compared to is built on the work reported in $[20,21]$ where a 5 th order state space model is constructed from a 1st order Fourier series with no parasitic capacitors included.

Table III shows the measured DC output voltage of the prototype converter, and the calculated DC output voltage for the two models with the difference from the voltage measured on the prototype, $\Delta V$. Inspecting the table, it is found that the proposed model is closer to the measured results than what has been accomplished with the prior art. The proposed model has its steady state output voltage within $1.1 \%$ of the measured output voltage, while the prior art is within $8.5 \%$.

Fig. 8 shows the measured and calculated frequency responses from the switching frequency to the output voltage of the converter. Observing the figure, we find a strong correlation between the proposed model and the implemented prototype up to $600 \mathrm{kHz}$ from which the magnitude of the measurement increases due to the resonance and switching frequency. Thus the measurements diverge from the model that is no longer valid due to the exceeding of the Nyquist frequency. The measured frequency response has a DC-gain of $-60.6 \mathrm{~dB}$, followed by a first order low pass filter response at $5.9 \mathrm{kHz}$, followed again by the beat frequency which causes a

TABLE III: DC output voltage of the resonant converter in steady state for the prototype (measured) and the two models (calculated).

\begin{tabular}{c|ccc}
\hline & Prototype & Proposed & Prior Art \\
\hline$V_{O_{D C}}$ & $210 \mathrm{~V}$ & $212.4 \mathrm{~V}$ & $192.2 \mathrm{~V}$ \\
$\Delta V$ & & $2.4 \mathrm{~V}(1.1 \%)$ & $-17.8 \mathrm{~V}(-8.5 \%)$ \\
\hline
\end{tabular}

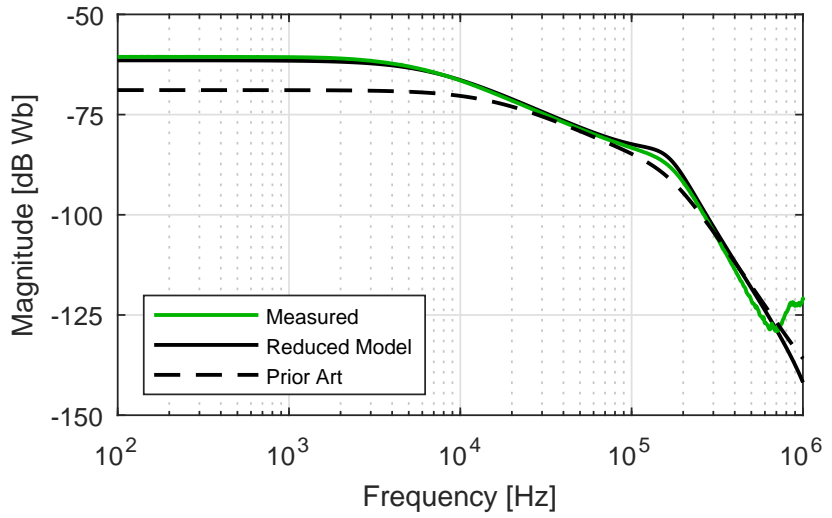

Fig. 8: Frequency response for $V_{o} / f_{s w}$ measured and calculated for the reduced model.

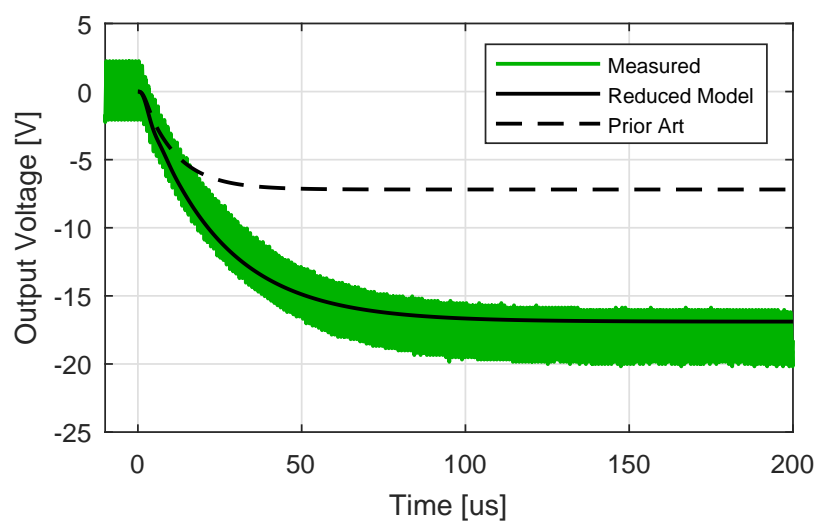

Fig. 9: Step response for $V_{o} / f_{s w}$ measured and calculated for the reduced model. All signals are offset to zero, and the step goes from $1.01 \mathrm{MHz}$ to $1.03 \mathrm{MHz}$.

second order low pass filter response at about $162 \mathrm{kHz}$. The DC-gain of the proposed model is off by $0.9 \mathrm{~dB}$, and the model matches the damping and cutoff frequencies of the two low pass filter responses. The small observed differences between the proposed model and the measurements are caused by minor discrepancies in the expected and actual component values leading to a slight difference in the response. On the other hand, the prior art is off by $8.3 \mathrm{~dB}$, and has the cutoff frequency of the first low pass filter at $15.8 \mathrm{kHz}$. However, the location of the beat frequency matches the measurement but the response is too damped.

Fig. 9 shows the response of the output voltage for the prototype converter and the two models when stepping the switching frequency, $f_{s w}$, up $20 \mathrm{kHz}$. The increase in frequency moves the switching frequency further away from the resonance frequency $f_{r}$, causing a voltage reduction on the output. The output voltage of the prototype falls by 18 $\mathrm{V}$ and settles to within $2 \%$ of the final voltage after about $100 \mu$ s with no overshoot. The proposed model has a smaller step of $-16.9 \mathrm{~V}$ and a faster settling time of about $91 \mu \mathrm{s}$. Meanwhile the prior art steps to $-7.2 \mathrm{~V}$ with a settling time of $41 \mu \mathrm{s}$. Hence the gains of the models for the steps correspond to the gain differences found in the frequency response in Fig. 8. 


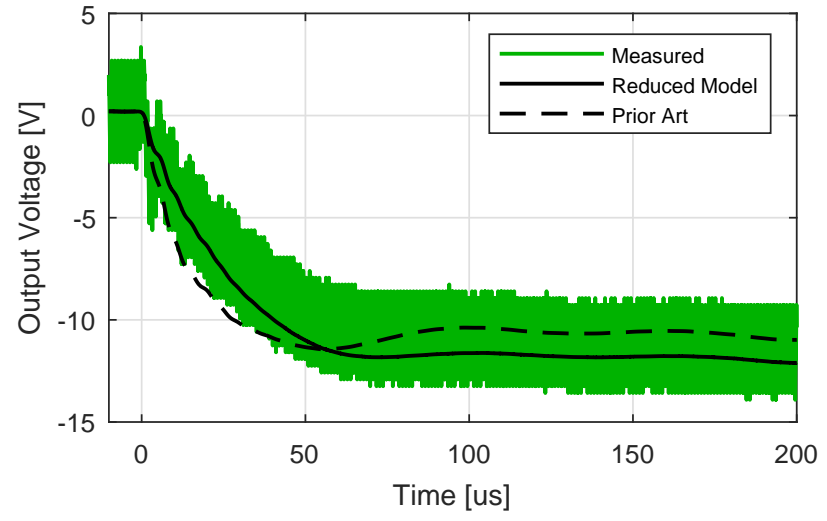

Fig. 10: Response for a $20 \mathrm{~V}$ step in the supply voltage measured and calculated for the reduced model. All signals are offset to zero, and the step steps from $348 \mathrm{~V}$ to $328 \mathrm{~V}$.

Lastly, Fig. 10 shows a $20 \mathrm{~V}$ step in the power supply for the prototype and the two models. The step was conducted by shorting out a $20 \mathrm{~V}$ supply hence creating a sudden drop in the supply voltage. However, the lab induced voltage drop contains damped oscillations that influence the step behavior. To obtain the best possible comparison between the models and the prototype, the actual voltage drop was logged and used as the step for the models. Observing Fig. 10 we see that the $20 \mathrm{~V}$ step causes the output of the converter to fall with $11.3 \mathrm{~V}$ in a well damped fashion. Both of the models also fall to approximately the same value with the prior art falling to $-10.8 \mathrm{~V}$ and the proposed model $-11.8 \mathrm{~V}$. However, the transient of the proposed model follows the measured response more precisely than the prior art which falls faster and has a slight undershoot.

To finalize this section, Table IV summarizes the results from the conducted measurements. From the table it is found that the proposed model is closer to the prototype converter for all the measurement. Especially the signal path from $f_{s w}$ to the output receives a significant improvement with the proposed model. The DC-gain error is improved with $7.4 \mathrm{~dB}$ and the error in the low frequency pole, $f_{l p}$ is reduced to less than $16.9 \%$ from $168 \%$ when comparing the prior art to the proposed reduced model.

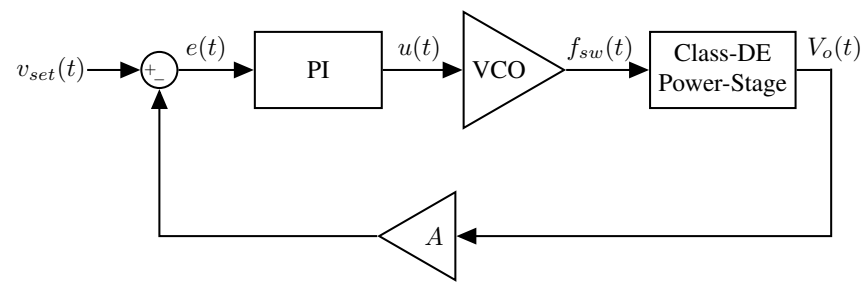

Fig. 11: Block diagram of the closed loop configuration of the class-DE converter.
TABLE IV: Summary of the measurement results.

\begin{tabular}{r|ccc}
\hline Model & Prototype & Proposed & Prior Art \\
\hline Steady State & & & \\
$V_{o_{D} C}$ & $210 \mathrm{~V}$ & $212.4 \mathrm{~V}$ & $192.2 \mathrm{~V}$ \\
$\Delta V$ & & $2.4 \mathrm{~V}(1.1 \%)$ & $-17.8 \mathrm{~V}(-8.5 \%)$ \\
& & & \\
Frequency Response & & & $-68.9 \mathrm{~dB}$ \\
DC-Gain & $-60.6 \mathrm{~dB}$ & $-61.5 \mathrm{~dB}$ & $15.8 \mathrm{kHz}$ \\
$f_{l p}$ & $5.9 \mathrm{kHz}$ & $6.9 \mathrm{kHz}$ & $169 \mathrm{kHz}$ \\
$f_{\text {beat }}$ & $162 \mathrm{kHz}$ & $166 \mathrm{kHz}$ & \\
& & & $41 \mu \mathrm{s}$ \\
Step Response $\left(f_{s w}\right)$ & $100 \mu \mathrm{s}$ & $91 \mu \mathrm{s}$ & $-7.2 \mathrm{~V}$ \\
Settling time & $100.8 \mathrm{~V}(60 \%)$ \\
Final value & $-18 \mathrm{~V}$ & $-16.9 \mathrm{~V}$ & \\
$\Delta V$ & & $1.1 \mathrm{~V}(6.1 \%)$ & $10.8 \mathrm{~V} \%)$ \\
Step Response $\left(V_{s}\right)$ & & $-11.8 \mathrm{~V}$ & $-10.8 \mathrm{~V}(4.4 \%)$ \\
Final value & $-11.3 \mathrm{~V}$ & $-0.5 \mathrm{~V}(-4.4 \%)$ & $0.5 \mathrm{~V} \%$ \\
$\Delta V$ & & &
\end{tabular}

\section{CONTROL EXAMPLE}

This section presents the design of a PI-controller for the SRC Class-DE converter based on the proposed model and prior art. The closed-loop responses of the two controllers are compared to assess the improvement by the proposed model.

\section{A. Design}

The objective of the controllers is to regulate the output voltage of the converter by adjusting the switching frequency. Fig. 11 shows the closed loop configuration of the converter where $A$ is an attenuation factor of $1 / 70$. To obtain comparable controllers for the two models, numerical optimization is utilized. The numerical optimization is set to minimize the Integral Absolute Error (IAE) of the output, and the Integral Square Error (ISE) of the control signal by changing the integral and proportional gain of the PI-controller. Equation (15) shows the cost function.

$$
J=\int\left|V_{\text {ref }}-V_{o}(t)\right|+R\left(u_{\text {ref }}-u(t)\right)^{2} \mathrm{~d} t
$$

In (15), $V_{o}(t)$ is the change in the voltage output of the converter due to a step in the frequency, and $V_{r e f}$ is the reference output voltage. In this case, the DC-gain of the model. Likewise, $u(t)$ is the control signal from the PI-controller and $u_{r e f}$ is the final value after the step. Lastly, $R$ is a tuning parameter used to control how aggressive the response should be. $R$ is selected to be 0.01 which results in a fast response with a limited overshoot in $u(t)(<10 \%)$ for both models. Running the optimization results in the controller parameters found in Table $\mathrm{V}$, where $J^{*}$ is the final cost.

TABLE V: Controller parameters

\begin{tabular}{r|ccc}
\hline Model & $K_{p}$ & $K_{i}$ & $J^{*}$ \\
\hline Proposed & 1.24 & 61290 & 0.8443 \\
Prior Art & 2.66 & 274392 & 0.2384 \\
\hline
\end{tabular}

From Table V, we find that the controller designed on the prior art model is more aggressive and has a lower final cost compared to the controller designed on the proposed model. 


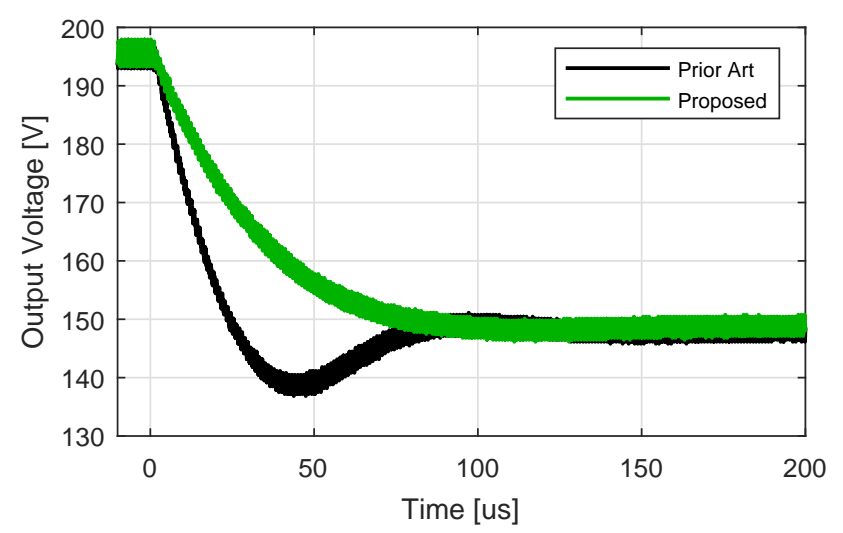

(a) Output voltage $y(t)$

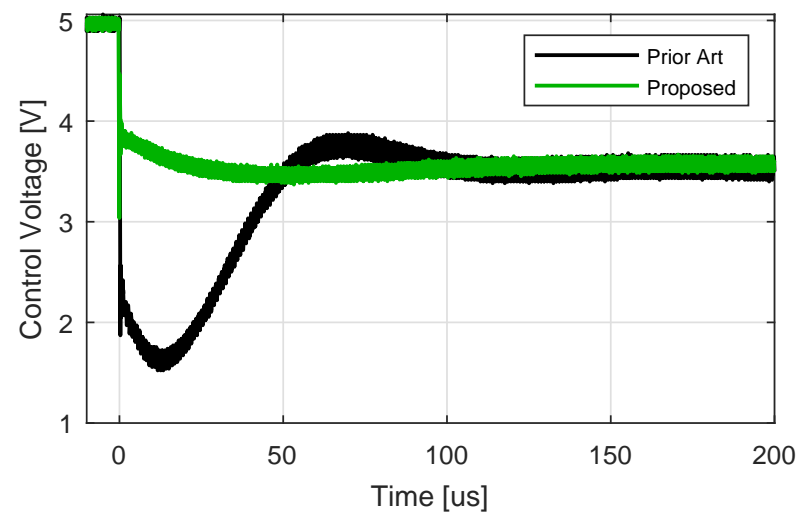

(b) Control voltage $u(t)$

Fig. 12: Closed-Loop step response of the implemented converter with the controller designed with the proposed model and the prior art. (a) shows the movement in the output voltage, and (b) the control signal from the PI-controller.

This suggest that the prior art controller should provide a faster response with less overshoot in the control signal. However, based on the findings in Section V-B this will not be the case when tested on the prototype.

\section{B. Closed-Loop Step Response}

To verify the performance of the two designed controllers, the controllers are connected to the prototype resonant converter. A step of $1 \mathrm{~V}$ on the reference, equating to $50 \mathrm{~V}$ on the output, is applied. Fig. 12 shows the resulting steps for the two controllers. Fig. 12a shows the output voltage and Fig. 12b shows the control signal from the PI-controller.

The responses in Fig. 12 clearly shows that the overshoot for the controller based on the prior art is significantly larger than the expected $(<10 \%)$, indicating that the model is not representative for the actual converter. The controller based on the prior art has a faster rise time on the output than the controller based on the proposed model. However, the faster rise time results in an overshoot, and the settling time for both controllers ends up being about $90 \mu$ s. The response of the system with the controller based on the proposed model behaves similar to what is expected from the control design, indicating that the proposed model is a good representation of the actual converter. The initial overshoot observed in the control signal in Fig. 12b at $t=0$ is caused by a large ground loop creating high-frequency oscillations.

Finally, Fig. 13 shows the measured and expected overshoots in the output voltage and the control signal for both of the controllers. From the figure, more than 40 times difference in the overshoot in the control signal of the prior art is evident, while almost no change is observed between the expected overshoot and the measured overshoot for the system based on the proposed model.

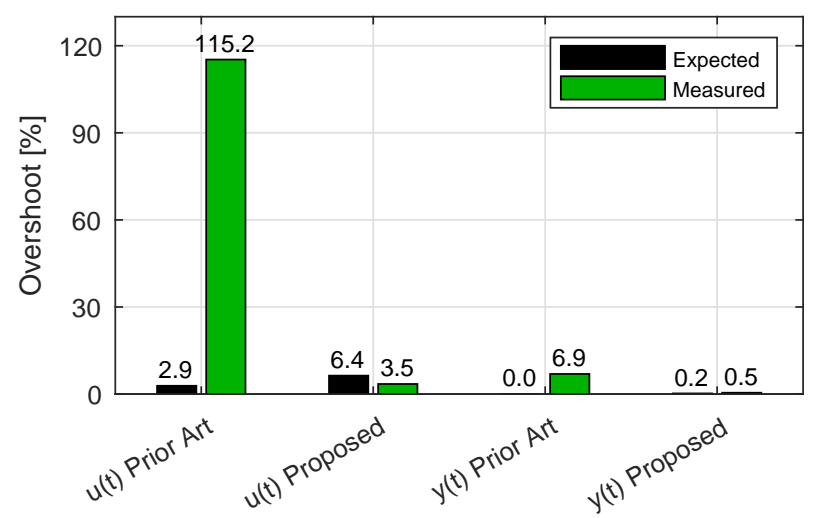

Fig. 13: Comparison of expected and measured overshoot for both models. The expected value for $y(t)$ prior art is zero and the overshoot for both the expected and measured $y(t)$ for the proposed model is less than $1 \%$.

\section{CONCLUSION}

This paper presents an improved model for class-DE series resonant converters operating at high frequencies. It is identified that the parasitic capacitances of the switching devices in the converter have a significant impact on the converter behavior and output voltage, and should be included in models once high frequency operation is considered. Using the generalized averaging modeling technique, a model incorporating parasitics is derived. The model is a high order model since multiple harmonics need to be considered to capture the influence of the parasitic components adequately. To mitigate the difficulties of working with high order models, a reduction method that retains all the relevant dynamics is presented. The reduced model is compared against a prototype class-DE SRC as well as prior art, and it is found that the reduced model is able to model the prototype accurately. The gain error is improved with more than $7 \mathrm{~dB}$, and the error in the low frequency pole is reduced to less than $16.9 \%$ from $168 \%$ when comparing the proposed reduced model with the prior art. A PI-controller is designed for the prior art and the proposed model, and tested on the prototype. It is found that the desired control behavior and the measured behavior matched for the proposed model while the prior art resulted in more than 40 times larger overshoot in the measurements compared to what was expected from the prior art model, showing the discrepancy that exists between the prior art model and the measurement. 


\section{APPENDIX}

The Appendix presents the equations used to calculate the coefficients for the Fourier series used in this work. To shorten the equations, the following substitution is applied:

$$
C_{b}(t)=\int I_{r}(t) \mathrm{d} t
$$

A. Fourier series for the voltage node $V_{h b}$

$$
\begin{aligned}
a_{V_{h b}}= & \frac{1}{\pi C_{d} \omega}\left(\int_{-\phi}^{t_{o n}-\phi} C_{b}(t) \sin (n t) \mathrm{d} t\right. \\
& \left.-C_{b}(-\phi) \int_{-\phi}^{t_{o n}-\phi} \sin (n t) \mathrm{d} t\right) \\
& +\frac{1}{\pi} \int_{t_{o n}-\phi}^{t_{o n}+\pi-\phi} V_{o}(t) \sin (n t) \mathrm{d} t \\
b_{V_{h b}}= & \frac{1}{\pi C_{d} \omega}\left(\int_{-\phi}^{t_{o n}-\phi} C_{b}(t) \cos (n t) \mathrm{d} t\right. \\
& \left.-C_{b}(-\phi) \int_{-\phi}^{t_{o n}-\phi} \cos (n t) \mathrm{d} t\right) \\
& +\frac{1}{\pi} \int_{t_{o n}-\phi}^{t_{o n}+\pi-\phi} V_{o}(t) \cos (n t) \mathrm{d} t \\
b_{0}= & \frac{V_{o_{D C}}}{2}
\end{aligned}
$$

Valid for: $n=1,3, \ldots N$.

\section{B. Fourier series for the current $I_{h b}$}

$$
\begin{aligned}
a_{I_{h b}} & =\frac{1}{\pi} \int_{t_{o n}-\phi}^{\pi-\phi} I_{r}(t) \sin (n t) \mathrm{d} t \\
b_{I_{h b}} & =\frac{1}{\pi} \int_{t_{o n}-\phi}^{\pi-\phi} I_{r}(t) \cos (n t) \mathrm{d} t \\
b_{0} & =\frac{1}{2 \pi} \int_{t_{o n}-\phi}^{\pi-\phi} I_{r}(t) \mathrm{d} t
\end{aligned}
$$

Valid for: $n=1,2, \ldots N$.
C. Fourier series for the voltage node $V_{s w}$

$$
\begin{aligned}
a_{V_{s w}=} & \frac{1}{\pi}\left(\frac{-1}{C_{o s s} \omega} \int_{0}^{\pi(1-2 D)} C_{b}(t) \sin (n t) \mathrm{d} t\right. \\
& +\left(\frac{C_{b}(0)}{C_{o s s} \omega}-V_{s}\right) \int_{0}^{\pi(1-2 D)} \sin (n t) \mathrm{d} t \\
& \left.+V_{s} \int_{\pi(1-2 D)}^{\pi} \sin (n t) \mathrm{d} t\right) \\
b_{V_{s w}}= & \frac{1}{\pi}\left(\frac{-1}{C_{o s s} \omega} \int_{0}^{\pi(1-2 D)} C_{b}(t) \cos (n t) \mathrm{d} t\right. \\
& +\left(\frac{C_{b}(0)}{C_{o s s} \omega}-V_{s}\right) \int_{0}^{\pi(1-2 D)} \cos (n t) \mathrm{d} t \\
& \left.+V_{s} \int_{\pi(1-2 D)}^{\pi} \cos (n t) \mathrm{d} t\right) \\
b_{0}= & 0
\end{aligned}
$$

Valid for: $n=1,3, \ldots N$.

\section{REFERENCES}

[1] D. J. Perreault, J. Hu, J. M. Rivas, Y. Han, O. Leitermann, R. C. N. Pilawa-Podgurski, A. Sagneri, and C. R. Sullivan, "Opportunities and Challenges in Very High Frequency Power Conversion," in 2009 Twenty-Fourth Annual IEEE Applied Power Electronics Conference and Exposition, pp. 1-14, Feb 2009.

[2] A. Knott, T. M. Andersen, P. Kamby, J. A. Pedersen, M. P. Madsen, M. Kovacevic, and M. A. Andersen, "Evolution of very high frequency power supplies," IEEE Journal of Emerging and Selected Topics in Power Electronics, vol. 2, no. 3, pp. 386-394, 2014.

[3] J. M. Rivas, D. Jackson, O. Leitermann, A. D. Sagneri, Y. Han, and D. J. Perreault, "Design considerations for very high frequency dc-dc converters," in Power Electronics Specialists Conference, 2006. PESC'06. 37th IEEE, pp. 1-11, IEEE, 2006.

[4] N. Bertoni, G. Frattini, R. G. Massolini, F. Pareschi, R. Rovatti, and G. Setti, "An Analytical Approach for the Design of Class-E Resonant DC-DC Converters," IEEE Transactions on Power Electronics, vol. 31, no. 11, pp. 7701-7713, 2016.

[5] X. Gao, H. Wu, and Y. Xing, "A Multioutput LLC Resonant Converter With Semi-Active Rectifiers," IEEE Journal of Emerging and Selected Topics in Power Electronics, vol. 5, no. 4, pp. 1819-1827, 2017.

[6] M. Noah, S. Endo, H. Ishibashi, K. Nanamori, J. Imaoka, K. Umetani, and M. Yamamoto, "A Current Sharing Method Utilizing Single Balancing Transformer for a Multiphase LLC Resonant Converter With Integrated Magnetics," IEEE Journal of Emerging and Selected Topics in Power Electronics, vol. 6, no. 2, pp. 977-992, 2018.

[7] H. Ma, Y. Li, Q. Chen, L. Zhang, and J. Xu, "A Single-Stage Integrated Boost-LLC AC-DC Converter 
With Quasi-Constant Bus Voltage for Multichannel LED Street-Lighting Applications," IEEE Journal of Emerging and Selected Topics in Power Electronics, vol. 6, no. 3, pp. 1143-1153, 2018.

[8] M. K. Kazimierczuk and D. Czarkowski, Resonant power converters. John Wiley \& Sons, 2012.

[9] C. Nwosu and M. Eng, "State-space averaged modeling of a nonideal boost converter," The pacific journal of science and Technology, vol. 2, no. 9, pp. 1-7, 2008.

[10] S. R. Sanders and G. C. Verghese, "Synthesis of averaged circuit models for switched power converters," IEEE Transactions on Circuits and systems, vol. 38, no. 8, pp. 905-915, 1991.

[11] K. Mandal, S. Banerjee, C. Chakraborty, and M. Chakraborty, "Bifurcations in frequency controlled load resonant DC-DC converters," in 2012 IEEE International Symposium on Circuits and Systems, pp. 1135-1138, IEEE, 2012.

[12] O. Dranga, B. Buti, and I. Nagy, "Stability analysis of a feedback-controlled resonant DC-DC converter," IEEE Transactions on Industrial Electronics, vol. 50, no. 1, pp. 141-152, 2003.

[13] P. C. Luk, S. Aldhaher, W. Fei, and J. F. Whidborne, "State-Space Modeling of a Class $E^{2}$ Converter for Inductive Links," IEEE Transactions on Power Electronics, vol. 30, pp. 3242-3251, June 2015.

[14] J. Kwon, X. Wang, F. Blaabjerg, C. L. Bak, A. R. Wood, and N. R. Watson, "Linearized modeling methods of AC-DC converters for an accurate frequency response," IEEE Journal of Emerging and Selected Topics in Power Electronics, vol. 5, no. 4, pp. 1526-1541, 2017.

[15] R. Z. Scapini, L. V. Bellinaso, and L. Michels, "Stability analysis of half-bridge rectifier employing LTP approach," in IECON 2012-38th Annual Conference on IEEE Industrial Electronics Society, pp. 780-785, IEEE, 2012.

[16] G. N. Love and A. R. Wood, "Harmonic state space model of power electronics," in 2008 13th International Conference on Harmonics and Quality of Power, pp. 1-6, IEEE, 2008.

[17] V. Salis, A. Costabeber, S. M. Cox, and P. Zanchetta, "Stability assessment of power-converter-based ac systems by LTP theory: eigenvalue analysis and harmonic impedance estimation," IEEE Journal of Emerging and Selected Topics in Power Electronics, vol. 5, no. 4, pp. 1513-1525, 2017.

[18] V. Salis, A. Costabeber, S. M. Cox, A. Formentini, and P. Zanchetta, "Stability Assessment of High-Bandwidth DC Voltage Controllers in Single-Phase Active Front Ends: LTI Versus LTP Models," IEEE Journal of Emerging and Selected Topics in Power Electronics, vol. 6, no. 4, pp. 2147-2158, 2018.

[19] S. Sanders and J. Noworolski, "Generalized averaging method for power conversion circuits," IEEE Transactions on Power Electronics, vol. 6, no. 2, 1991.

[20] M. F. Menke, Á. R. Seidel, and R. V. Tambara, "LLC LED Driver Small-Signal Modeling and Digital Control Design for Active Ripple Compensation," IEEE Transactions on Industrial Electronics, vol. 66, no. 1, pp. 387-396, 2019.
[21] P. Wang, C. Liu, and L. Guo, "Modeling and simulation of full-bridge series resonant converter based on generalized state space averaging," in Applied Mechanics and Materials, vol. 347, pp. 1828-1832, Trans Tech Publ, 2013.

[22] C. Buccella, C. Cecati, H. Latafat, P. Pepe, and K. Razi, "Observer-Based Control of LLC DC/DC Resonant Converter Using Extended Describing Functions," IEEE Transactions on Power Electronics, vol. 30, no. 10, pp. 5881-5891, 2015.

[23] J. Groves, "Small-signal analysis using harmonic balance methods," in Power Electronics Specialists Conference, 1991. PESC'91 Record., 22nd Annual IEEE, pp. 74-79, IEEE, 1991.

[24] S. Skogestad and I. Postlethwaite, Multivariable feedback control: analysis and design, vol. 2. Wiley New York, 2007.

[25] P. Benner, "Numerical linear algebra for model reduction in control and simulation," GAMM-Mitteilungen, vol. 29, no. 2, pp. 275-296, 2006.

[26] GaNSystems, "GaN transistor GS66502B," 2017.

[27] GeneSiC, "Silicon Carbide Power Schottky Diode GB01SLT06-214," 2014.

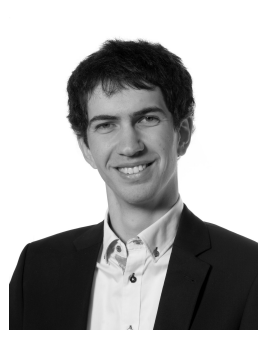

Nicolai J. Dahl Nicolai J. Dahl (S'19) is a Ph.D student at the electronics group at the Technical University of Denmark, Kongens Lyngby, Denmark. He received both his B.Sc. degree and M.Sc. degree from the Technical University of Denmark in June 2016 and August 2018 respectively. His research interest include control theory, system modeling, signal processing, and optimization, which he has applied in the fields of switch-mode power amplifiers, resonant converters, and his current research topic, time-based control.

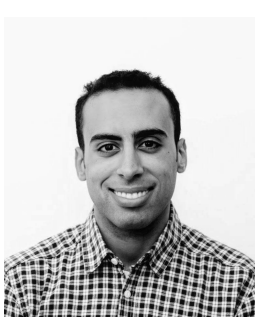

Ahmed M. Ammar Ahmed M. Ammar (S'11) received his B.Sc. and M.Sc. degrees in electrical engineering from Mansoura University and Nile University, Egypt, in 2011 and 2014, respectively. His experience includes positions at different industries including Intel Corporation, Imec, and Mentor Graphics (now Mentor, a Siemens Business). He is currently working as a graduate research and teaching assistant at the Department of Electrical Engineering in the Technical University of Denmark towards his Ph.D. Degree. His current research interests include power electronics, integrated power converters, and power management and delivery circuits and systems.

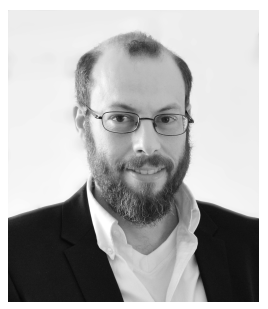

Arnold Knott Arnold Knott (M'10) received the DiplomIngenieur $(\mathrm{FH})$ degree from the University of Applied Sciences in Deggendorf, Germany, in 2004. From 2004 until 2009 he has been working with Harman/Becker Automotive Systems GmbH in Germany and USA, designing switch-mode audio power amplifiers and power supplies for automotive applications. In 2010 he earned the Ph.D. degree from the Technical University of Denmark, Kongens Lyngby, Denmark working on a research project under the title "Improvement of out-of band Behavior in Switch-Mode Amplifiers and Power Supplies by their Modulation Topology". From 2010 to 2013 he was Assistant Professor and since 2013 Associate Professor at the Technical University of Denmark. His interests include switchmode audio power amplifiers, power supplies, active and passive components, integrated circuit design, acoustics, radio frequency electronics, electromagnetic compatibility and communication systems. 


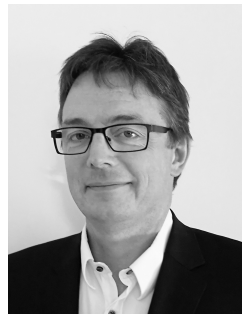

Michael A. E. Andersen Michael A. E. Andersen (M'88) received the M.Sc.E.E. and Ph.D. degrees in power electronics from the Technical University of Denmark, Kongens Lyngby, Denmark, in 1987 and 1990, respectively. He is currently a Professor of power electronics at the Technical University of Denmark, where since 2009, he has been the Deputy Head of the Department of Electrical Engineering. $\mathrm{He}$ is the author or coauthor of more than 300 publications. His research interests include switchmode power supplies, piezoelectric transformers, power factor correction, and switch-mode audio power amplifiers. 